\title{
Evaluación del logro del perfil de egreso. Un estudio de caso
}

\section{Evaluation of the achievement of the graduation profile. A case study}

Celia Carrera Hernández ${ }^{1}$

Yolanda Isaura Lara García ${ }^{2}$

Josefina Madrigal Luna ${ }^{3}$

\section{Resumen}

La presente investigación cualitativa se abordó desde la fenomenología con un estudio de caso, con el objetivo de evaluar de manera comprensiva el perfil de egreso de la Licenciatura en Intervención Educativa (LIE) que oferta la Universidad Pedagógica Nacional del Estado de Chihuahua (UPNECH) a partir de la experiencia de los alumnos que cursan el octavo semestre de la LIE y la percepción de sus profesores. Se aplicaron técnicas como la observación, entrevista y encuesta. Participaron50 estudiantes de la generación 20013-2018 y 10 profesores que les impartieron cursos durante su proceso de formación. Se abordó la evaluación de programas con enfoque receptivo-comprensivo (Stake, 2007) a partir de la reflexión sobre la relación currículo-sociedad. La mirada epistémica fue crítica para identificar desde un ejercicio de autorreflexión las barreras que afectaron el logro del

\footnotetext{
${ }^{1}$ Celia Carrera Hernández. Profesora-investigadora de la Universidad Pedagógica Nacional del Estado de Chihuahua, Campus Chihuahua, México. Pertenece al Sistema Nacional de Investigadores nivel I y cuenta con Perfil PRODEP. Correo electrónico: ccarrera@upnech.edu.mx

ID: http://orcid.org/0000-0002-2444-2204
}

${ }^{2}$ Yolanda Isaura Lara García. Profesora-investigadora de la Universidad Pedagógica Nacional del Estado de Chihuahua, Campus Parral, México. Cuenta con Perfil PRODEP y ha publicado varios libros. Correo electrónico: ylara@upnech.edu.mx ID: http://orcid.org/0000-0002-5250-9517

${ }^{3}$ Josefina Madrigal Luna. Profesora-investigadora de la Universidad Pedagógica Nacional del Estado de Chihuahua, Campus Parral, México. Es miembro del Sistema Nacional de Investigadores, nivel I, y cuenta con Perfil PRODEP. Correo electrónico: jmadrigal@upnech.edu.mx

ID: http://orcid.org/0000-0003-2190-3164 
perfil de egreso y los aspectos positivos que identificaron como favorecedores. Se encontró que la principal barrera para el logro del perfil de egreso es la docencia ya que los alumnos dominan los contenidos pero no asumen una postura reflexiva respecto a su proceso de formación, a los logros obtenidos y sobre todo a su papel como transformador social. Por lo que la universidad requiere generar espacios abiertos de diálogo al interior de la misma que recupere las valoraciones, percepciones y experiencias de profesores y alumnos con el fin de reorientar los procesos de implementación del currículo y el rediseño del mismo.

\title{
Palabras clave
}

Perfil de egreso, currículum, educación superior, evaluación.

\begin{abstract}
The present investigation of qualitative was approached from the phenomenology with a case study, with the objective of comprehensively evaluating the graduation profile of the Degree in Educational Intervention (LIE) offered by the National Pedagogical University of the State of Chihuahua (UPNECH ) based on the experience of the students who attend the eighth semester of the LIE and the perception of their teachers. Techniques such as observation, interview and survey were applied. Participants were 50 students from the 20013-2018 generation and 10 teachers who gave them courses during their training process. The evaluation of programs with a receptive-comprehensive approach (Stake, 2007) was considered based on the reflection on the relationship curriculum-society. The epistemic view was critical to identify from an exercise of self-reflection the barriers that affected the achievement of the graduation profile and the positive aspects that they identified as favoring. It was found that the main barrier to achieving the graduation profile is teaching since students dominate the content but do not assume a reflective stance regarding their training process, the achievements obtained and especially their role as a social transformer. For this reason, the university needs to generate open spaces of dialogue within the university to recover the evaluations, perceptions and experiences of professors and students in order to reorient the processes of curriculum implementation and the redesign of the same.
\end{abstract}

\section{Keywords}

Graduation profile, curriculum, higher education, curriculum, evaluation. 


\section{Introducción}

Una de las tareas de las instituciones educativas es la evaluación curricular que de manera sistemática provee información importante para realizar mejoras sustantivas a las mismas (Stufflebeam y Achinkfield, 1995). Desde esta perspectiva la evaluación es una fuerza positiva que sirve para identificar puntos débiles y fuertes con la intención de mejorar cuyas bases de valoración son las experiencias y percepciones de los usuarios.

La evaluación curricular implica el análisis del vínculo educaciónsociedad a partir del cual se problematiza el sentido de impertinencia de los programas y la formación de los futuros profesionistas (Del Bosto, 2015). Sin embargo, las universidades en el contexto internacional han implementado innovaciones curriculares en el diseño de los programas incorporando nuevos enfoques y metodologías pero ha faltado una evaluación que evidencie el logro del perfil de egreso en cuanto a su impertinencia respecto a las características de la sociedad actual. Para Barnett (1994) la pertinencia es la adaptación de la formación a los requerimientos del mercado; desde esta perspectiva se aprecia desconexión de la responsabilidad del currículo con la transformación social. Por otra parte, menciona a la impertinencia como el llamado a construir un pensamiento crítico que interrogue las estructuras de poder de la sociedad y busque la transformación de la vida de diferentes grupos sociales.

Los estudios recientes sobre el perfil de egreso buscan responder a la exigencia del mercado laboral a partir de identificar la pertinencia de la formación.

A partir de lo anterior, existe la necesidad de contar con mecanismos para evaluar, el logro del perfil de egreso de los alumnos de LIE con la recuperación de la experiencia de docentes y alumnos de manera reflexiva orientada a la búsqueda de formas innovadoras que incidan en cambios de carácter social en el campo de la educación, es decir, desde la impertinencia.

La LIE es un programa que oferta la Universidad Pedagógica Nacional del Estado de Chihuahua (UPNECH), e inició sus operaciones a partir del año 2002 con el objetivo de formar un profesional de la educación capaz de desempeñarse en diversos ámbitos educativos, a través de la adquisición de las competencias generales (propias de cualquier profesional del campo de la educación) y específicas (las adquiridas a través de las diferentes líneas profesionalizantes detectadas), que le permitan transformar la realidad educativa por medio de procesos de intervención. En este caso las competencias generales corresponden al perfil de egreso (UPN, 2002). Hasta el momento se desconoce la forma en la que se implementa el programa de LIE, dado que no se ha realizado un trabajo sistemático de 
evaluación que incluya la revisión de la impertinencia del perfil de egreso de los estudiantes de manera interna.

Por lo anterior, es importante, que se busquen procedimientos eficaces para evaluar los programas educativos desde el logro del perfil de egreso ya que la revisión de su impertinencia es prioritaria debido a que el conocimiento, en este caso relacionado con la intervención y la educación, es cada vez es más amplio y las necesidades del contexto social diversas.

A partir de los nudos críticos mencionados arriba se identifica que el supuesto problematizador es que, la licenciatura no cuenta con procesos para evaluar la impertinencia del perfil de egreso y el logro efectivo de éste a partir de la experiencia de estudiantes y profesores. Por lo anterior, la pregunta de investigación es: ¿Cómo evalúan los profesores y alumnos de la Licenciatura en Intervención Educativa el perfil de egreso que se especifica en el programa?

Las subpreguntas de investigación son:

- ¿Cómo se autoevalúan los alumnos respecto al logro del perfil de egreso y que factores identifican como favorecedores o como barreras para el logro de los mismos?

- ¿Cuáles son las percepciones de alumnos y profesores respecto al logro del perfil de egreso considerando las necesidades de formación y las características del contexto actual?

- ¿Cómo evalúan los profesores el perfil de egreso respecto a su experiencia en la implementación del programa?

El objetivo de la investigación es evaluar el perfil de egreso de la Licenciatura en Intervención Educativa a partir de la experiencia de alumnos que cursan el octavo semestre de la LIE y sus profesores.

Los objetivos específicos son:

- Comprender la forma en la que los alumnos se autoevalúan respecto al logro del perfil de egreso y qué factores identifican como favorecedores o como barreras para el logro de los mismos.

- Analizar las percepciones de alumnos y profesores respecto al logro del perfil de egreso considerando las necesidades de formación y las características del contexto actual.

- Conocer la forma en la que los profesores evalúan el perfil de egreso respecto a su experiencia en la implementación del programa.

Se considera que esta investigación es importante ya que ofrece una mirada alternativa a la evaluación del perfil de egreso respecto a la forma racional en la que se revisa la pertinencia del mismo para que los alumnos logren insertarse en el ámbito laboral, pero se deja de lado la formación reflexiva y 
crítica con la intención de reconocer el entorno para buscar mejorarlo desde una postura epistemológica crítica.

\section{Fundamentos teóricos}

El enfoque teórico de evaluación curricular que se retoma es el comprensivo ya que busca analizar la formación del sujeto en su compromiso con la transformación de la sociedad, responsabilidad que se desprende del programa de la LIE desde el propósito para el que fue diseñado. Para Stenhouse (1991) el currículo es un elemento situado en un contexto social que expresa un proyecto de formación de estudiantes y que hace posible la construcción de un determinado tipo de sujeto de acuerdo a la sociedad. Desde esta perspectiva teórica no se busca evaluar el perfil de egreso para encontrar formas de satisfacer el ámbito laboral ya que limita una formación reflexiva de sus estudiantes, sino la postura desde la cual se asume la presente investigación se refiere a que el currículo debe responder a las exigencias del contexto social y ocuparse de la formación de estudiantes que aporten a la transformación social. Por ello, se habla de resignificar el vínculo currículo-sociedad en una cultura neoliberal que busca solamente formar para insertar en el ámbito laboral no para que reflexionen sobre su realidad y sean capaces de proponer y realizar cambios importantes en favor de la sociedad en general o de grupos específicos. El perfil de egreso es el punto de convergencia entre la formación y la sociedad.

El perfil de egreso es el "conjunto de conocimientos, competencias y actitudes que el/ la estudiante de la carrera o programa habrá internalizado al momento de su egreso" (CINDA, 2017, p. 4). La evaluación busca identificar la impertinencia del perfil de egreso desde ejes conceptuales como: desempeños notables situados para reflexionar sobre la realid ad en prácticas profesionales, los elementos discriminadores de contexto durante el proceso de formación y la transferencia crítica para reconocer la importancia de su formación en la transformación social.

La mirada epistémica es crítica porque identifica desde un ejercicio de autorreflexión las barreras para el logro del perfil de egreso y los aspectos positivos que identifican como favorecedores desde la percepción de profesores y alumnos, el proceso de formación, el desempeño durante las prácticas profesionales y la identificación de las necesidades de transformación. 
RECIE. Revista Electrónica Científica de Investigación Educativa Vol. 4, núm. 2, enero-diciembre 2019, pp. 1019-1028.

\section{Metodología}

El abordaje metodológico es del tipo fenomenológico porque recupera percepciones de profesores y estudiantes respecto al perfil de egreso del programa de la LIE que oferta la UPNECH ya que a través de la fenomenología se contribuye al "conocimiento de la realidad escolar, recuperando las vivencias de los actores del proceso formativo" (Aguirre y Jaramillo, 2012, p. 3) Se consideró estudio de caso intrínseco porque corresponde a la evaluación de un programa específicamente. Según Stake (2007) en el estudio de caso se revisa la particularidad y la complejidad de un caso singular, para llegar a comprender su actividad en circunstancias importantes. El objeto de estudio es el perfil de egreso para comprender su logro durante el proceso de implementación del programa.

Participaron 50 estudiantes y 10 profesores en el análisis de la impertinencia del perfil de egreso considerando las necesidades de formación de los estudiantes y las características del contexto social actual. Los datos se recuperaron a partir de la observación, entrevista semiestructurada y de un cuestionario mixto ya que según Embree (2003), la fenomenología orientada al análisis reflexivo identifica hechos observados, creencias, valoraciones y voliciones de los agentes educativos. El método de análisis de datos fue el inductivo.

\section{Resultados}

Los estudiantes se auto evalúan respecto al logro del perfil de egreso es la siguiente:

Figura 1. Autoevaluación de los estudiantes respecto al logro del perfil de egreso.

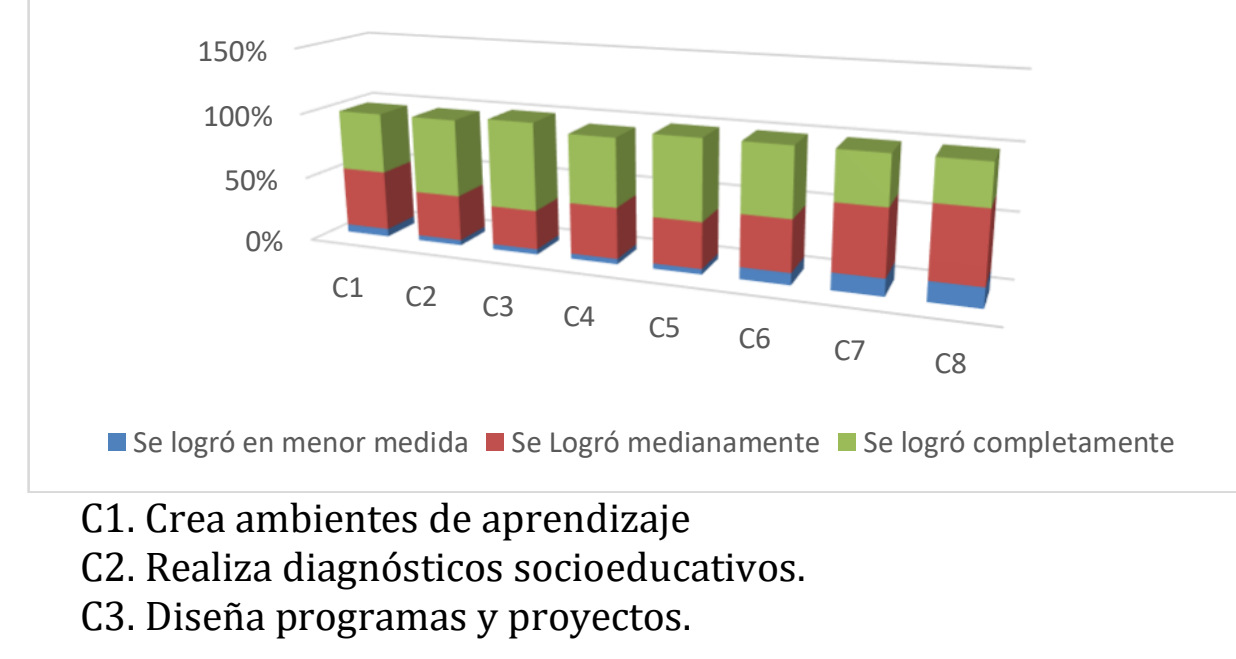

Carrera Hernández, C.; Lara García, Y.I.; y Madrigal Luna, J. 
C4. Asesora a individuos, grupos e instituciones.

C5. Planea procesos, acciones y proyectos.

C6. Identifica, desarrolla y adecúa proyectos.

C7. Evalúa instituciones, sujetos y procesos.

C8. Desarrolla procesos de formación.

Una de las competencias que se logran en menor medida se refiere a identificar, desarrollar y adecuar proyectos educativos que respondan a la resolución de problemáticas específicas con base en el conocimiento de diferentes enfoques pedagógicos, administrativos y de la gestión, organizando y coordinando los recursos para favorecer el desarrollo de las instituciones con responsabilidad y visión prospectiva (C6). Los alumnos reflexionan sobre la necesidad de indagar en diferentes instancias los proyectos existentes y con ello realizar adecuaciones de acuerdo al contexto y sus necesidades para poder implementarlos, pero critican la unidireccionalidad existente en la tarea de realizar diagnósticos para identificar los problemas, diseñar la intervención, implementarla y evaluar sus resultados.

Otra competencia que es considerada por los estudiantes como de bajo logro en menor medida es la de evaluar instituciones, procesos y sujetos tomando en cuenta enfoques, metodologías y técnicas de evaluación a fin de que le permitan valorar su pertinencia y generar procesos de retroalimentación, con una actitud crítica y ética (C7). Esta competencia es necesaria para el interventor ya que le posibilita proponer mejoras y transformar las instituciones educativas, los procesos que se desarrollan y los programas. Para el alumno la falta de vinculación entre la teoría y la práctica influye para que no se logre la competencia del curso e impacte en el logro del perfil de egreso.

La competencia que desde la percepción de profesores y alumnos de los alumnos no se logra es la de desarrollar procesos de formación permanente y promoverla en otros, con una actitud de disposición al cambio e innovación, utilizando los recursos científicos, tecnológicos y de interacción social que le permitan consolidarse como profesional autónomo (C8). Profesores y alumnos identifican la falta de reflexión y autocrítica a través de proceso de evaluación y autoevaluación constante para considerar la autoformación, el autodidactismo y la preocupación por participar en la formación de otros durante o al término de su formación en la universidad. Para los alumnos es necesaria la formación permanente mientras cursan el programa. Estas competencias impactan en la transformación social de forma decidida y debe promoverse al interior de las instituciones de manera constante. 
Las barreras para el logro del perfil de egreso son:

El ejercicio de una docencia universitaria de corte tradicional centrada en la tarea solamente del aula y orientada a contenidos teóricos sin incorporar experiencias de los estudiantes en situaciones reales para que puedan aplicar los conocimientos teóricos. Es decir la enseñanza no se sitúa en un contexto específico.

El contenido no es actualizado ni favorece la comprensión de los temas, por lo que se requiere el manejo de información reciente.

La falta de espacios formativos extracurriculares que impacten en el logro de las competencias como diplomados, invitación de expertos, talleres y conferencias.

Los factores favorecedores del logro del perfil de egreso fueron son: la oportunidad de vincular la teoría con la práctica en el espacio curricular de prácticas profesionales, desde la reflexión de los alumnos y poner en práctica los conocimientos adquiridos en cada uno de los cursos. Según Stenhouse (1991) la universidad debe tener un papel protagónico en la construcción de sociedad desde el currículo a partir de la relación teoríapráctica. Otro factor es el esfuerzo de actualización de los cursos que realizan algunos de los profesores a pesar de que no hay rediseño del programa e identifican la necesidad de mantener espacios de diálogo para reflexionar sobre el ejercicio de su práctica y la forma de mejorarla.

Para alumnos y profesores el perfil de egreso encamina al egresado a la transformación social dado que desarrollan habilidades para problematizar la realidad, planear e implementar soluciones así como valorar sus resultados en una sociedad que padece los efectos del neoliberalismo caracterizada por pobreza y violencia social principalmente.

El reclamo de los estudiantes es que se otorgue importancia al desarrollo de procesos de formación permanente resultado de espacios de diálogo entre alumnos y entre éstos y los profesores para que de manera constante emanen necesidades y propuestas de formación constante ya que como menciona Freire (2010), "aquél que se atreve a enseñar o intervenir jamás debe dejar de aprender."

\section{Discusión y conclusiones}

La formación del LIE es necesaria porque se orienta a la educación popular con una visión crítica de la realidad. Por ello, el perfil de egreso debe ser evaluado sistemáticamente para que la universidad logre un lugar protagónico en la construcción de una mejor sociedad desde el currículo, pues desde este último, es posible establecer una relación entre teoría y 
práctica, así como favorecer su implementación bajo una práctica pedagógica reflexiva y crítica, que desde las propuestas de profesores y alumnos aspira a lograr transformaciones que trasciendan la conducta y el conocimiento de sus egresados hacia el cambio de las comunidades a través de proyectos que respondan a las necesidades sociales.

Ante un currículo con orientación crítica, la revisión de la docencia se torna indispensable ya que puede convertirse en la barrera principal en el logro del perfil de egreso pues desde el currículo se demanda profesores críticos de su práctica docente, del progreso de los alumnos y de la realidad escolar. La formación del alumno depende en gran parte del currículo, pero en mayor medida de la percepción de los profesores sobre la formación de los interventores educativos y del ejercicio de su práctica, por lo que tienen una gran responsabilidad ante la sociedad. Por ello, al interior de las instituciones se debe favorecer la interacción permanente entre los actores educativos y la reflexión sobre el vínculo con una sociedad que se transforma constantemente (Del Basto y Ovalle, 2015).

\section{Referencias}

Aguirre, J.C. y Jaramillo, L.G. (2012). Aportes del método fenomenológico a la investigación educativa. Revista Latinoamericana de Estudios Educativos. No. 2. Vol. 8, pp.51-74. Manizales: Universidad de Caldas.

Barnett, R. (1994). Los límites de la competencia. Barcelona: Editorial Gedisa.

Centro Interuniversitario de Desarrollo (CINDA). (2017). Evaluación del logro de perfiles de egreso: experiencias universitarias. Chile: Grupo operativo coordinado por CINDA.

Del Basto y Ovalle, M. (2015). Una mirada crítica a la relación currículosociedad. Revista Latinoamericana de Estudios Educativos, No. 11. Vol 1. Pp. 111-127. Colombia.

Embree, Lester. (2003). Análisis reflexivo: Una primera introducción a la investigación fenomenológica. Reflective Analysis: A First Introduction into Phenomenological Investigation. Edición bilingüe, inglés/castellano. Morelia: Red Utopía / Jitanjáfora.

Freire, P. (2010). Cartas a quien se atreve a enseñar. 2ª Edición. Argentina: Siglo XXI editores.

Stenhouse, L. (1991). Investigación y desarrollo del currículum. Madrid: Morata.

Stufflebeam, D. y Achinkfield, A. (1995). Evaluación sistemática. Guía teórico-práctica. España: Centro de publicaciones del Ministerio de Educación y Ciencia, ediciones Paidós Ibérica. 
RECIE. Revista Electrónica Científica de Investigación Educativa Vol. 4, núm. 2, enero-diciembre 2019, pp. 1019-1028.

UPN (2002). Programa de reordenamiento de la oferta educativa de las unidades UPN. México: UPN. 